\section{Empfehlungen zur Sicherung der Qualität in der Bronchoskopie}

\author{
Arbeitsgruppe Qualitätssicherung in der Endoskopie \\ Sektion Endoskopie der Deutschen Gesellschaft für Pneumologie
}
K. Häussinger ${ }^{1}$, A. Ballin ${ }^{2}$,
H. D. Becker ${ }^{3}$, P. Bölcskei ${ }^{4}$,
R. Dierkesmann ${ }^{5}$, I. Dittrich ${ }^{6}$, W. Frank ${ }^{7}$, L. Freitag ${ }^{8}$, R. Gottschall ${ }^{9}$, W. R. Guschall ${ }^{6}$, W. Hartmann ${ }^{10}$, R. Hauck ${ }^{11}$, F. Herth ${ }^{3}$, D. Kirsten ${ }^{12}$, M. Kohlhäufl' ${ }^{1}$, A. Kreuzer ${ }^{13}$, R. Loddenkemper ${ }^{14}$, N. Macha ${ }^{8}$, A. Markus ${ }^{1}$, F. Stanzel', H. Steffen ${ }^{15}$, M. Wagner ${ }^{4}$

\author{
Recommendations for Quality Standards in Bronchoscopy. \\ Report of the Working Party on Recommendations for Quality Standards \\ in Endoscopy of the German Society of Pulmonology (Section Endoscopy)
}

Die Bronchoskopie ist die wichtigste Methode für die histologische, infektiologische und zelluläre Diagnostik von Atemwegsund Lungenerkrankungen. Sie wird überwiegend mit dem flexiblen Bronchoskop in Lokalanästhesie, seltener mit starren Instrumenten in Vollnarkose durchgeführt.

Die bronchoskopischen Basisverfahren umfassen die Inspektion, diagnostische Maßnahmen wie Gewinnung von Bronchialsekret zur zytologischen, mikrobiologischen und mykologischen Untersuchung, die Entnahme von Schleimhaut- und Lungengewebsproben, die bronchoalveoläre Lavage und therapeutische Manöver wie Absaugungen, Bougierungen und Entfernungen von Fremdkörpern. Diese Verfahren wurden in den letzten Jahren durch eine Reihe von technischen und methodischen Neuerungen zur Diagnostik (endobronchiale Sonographie und Fluoreszenzbronchoskopie) und Therapie (z.B. Laserkoagulation, photodynamische Therapie und Brachytherapie) ergänzt.

Empfehlungen zu diesen Spezialverfahren sind seitens der Deutschen Gesellschaft für Pneumologie bereits publiziert [1-3]. Auch von Gesellschaften des angloamerikanischen Sprachraums wurden vor kurzem Richtlinien hierzu veröffentlicht $[4,5]$.

\section{Zielsetzung und Methodik}

Ziel des vorliegenden Konzeptes ist es, die Qualität der pneumologischen Endoskopie im Rahmen der Patientenversorgung sicherzustellen und kontinuierlich zu verbessern. Die folgenden Empfehlungen sollen gleichermaßen für stationäre und ambulante Bedingungen gelten. Endoskopische Untersuchungen/Behandlungen sollen dabei

- zu vergleichbaren Ergebnissen in Diagnostik und Therapie führen,

- unter gleichen Sicherheitsmaßnahmen durchgeführt werden,

- gleiche Qualitätssicherungsmaßnahmen umfassen.

Den vorliegenden Empfehlungen zur Bronchoskopie ging eine bundesweite Erhebung zur Situation der Bronchoskopie in endoskopischen Abteilungen und Fachpraxen voraus, deren Daten unter anderem als Grundlage für eine „realistische“ Formulierung dieser Empfehlungen dienten [6]. Auch im angloamerikanischen Raum wurden Umfragen zur Situation der Bronchoskopie durchgeführt und publiziert [7-10]. Die erhobenen Daten zeigen, dass die Vorgehensweisen uneinheitlich und Empfehlungen bzw. Richtlinien erforderlich sind. Zur Ausarbeitung der vorliegenden

Institutsangaben

${ }^{1}$ Asklepios Fachkliniken München-Gauting, Zentrum für Pneumologie und Thoraxchirurgie

${ }^{2}$ Klinikum Leipzig

${ }^{3}$ Thoraxklinik Heidelberg-Rohrbach

${ }^{4}$ Klinikum Nürnberg, Medizinische Klinik 3 - Schwerpunkt Pneumologie

${ }^{5}$ Fachklinik Schillerhöhe, Gerlingen

${ }^{6}$ Lungenklinik Lostau, Zentrum für Pneumologie und Thoraxchirurgie

${ }^{7}$ Johanniter-Krankenhaus Treuenbrietzen, Klinik III, Fachklinik für Pneumologie

${ }^{8}$ Lungenklinik Hemer

${ }^{9}$ Klinik für Intensivtherapie und Anästhesiologie, Klinikum Jena

${ }^{10}$ Zentralkrankenhaus Bremen-Ost

${ }^{11}$ Städtisches Krankenhaus Bad Reichenhall

${ }^{12}$ Krankenhaus Großhansdorf, Zentrum für Pneumologie und Thoraxchirurgie

${ }^{13}$ Pulmologisches Zentrum Wien

${ }^{14}$ Lungenklinik Heckeshorn

${ }^{15}$ Pneumologische Facharztpraxis, Landsberg

Korrespondenzadresse

Prof. Dr. Karl Häußinger · Asklepios Fachkliniken München-Gauting ·

Robert Koch-Allee 2·82131 Gauting·E-mail: k.haeussinger@asklepios.com

Bibliografie

Pneumologie 2004; 58: 344-356 • c Georg Thieme Verlag KG Stuttgart · New York

DOI $10.1055 / \mathrm{s}-2004-818406$

ISSN 0934-8387 
Empfehlungen wurden zusätzlich die Leitlinien der American Thoracic Society [11], der British Thoracic Society [12], der Thoracic Society von Australien und Neuseeland [13] und die Empfehlungen der Kommission für Krankenhaushygiene und Infektionsprävention beim Robert-Koch-Institut herangezogen [14,15]. Soweit möglich liegen den Empfehlungen auch wissenschaftliche Daten zugrunde, die dem Leitlinienmanual der Arbeitsgemeinschaft der wissenschaftlichen medizinischen Fachgesellschaften (AWMF) [16] und der ärztlichen Zentralstelle für Qualitätssicherung in der Medizin (AZQ) [17] entsprechen und hinsichtlich ihrer Evidenz bewertet werden. Die Beurteilung der Aussagekraft erfolgt nach Evidenztypen, die in Evidenzgrade gewichtet werden (Tab.1).

Tab. 1 Bewertung der publizierten Literatur gemäß Aussagekraft nach Evidenztypen und Gewichtung in Empfehlungsgrade

\begin{tabular}{lll}
$\begin{array}{l}\text { Grad der } \\
\text { Empfehlung }\end{array}$ & Evidenz & \\
\hline A & I a & $\begin{array}{l}\text { Evidenz aufgrund von Meta-Analysen rando- } \\
\text { misierter, kontrollierter Studien }\end{array}$ \\
\hline A & I b & $\begin{array}{l}\text { Evidenz aufgrund mindestens einer randomi- } \\
\text { sierten, kontrollierten Studie }\end{array}$ \\
\hline B & II a & $\begin{array}{l}\text { Evidenz aufgrund mindestens einer gut ange- } \\
\text { legten kontrollierten Studie ohne Randomi- } \\
\text { sierung }\end{array}$ \\
\hline B & II b & $\begin{array}{l}\text { Evidenz aufgrund mindestens einer gut ange- } \\
\text { legten quasi experimentellen Studie }\end{array}$ \\
\hline Evidenz aufgrund gut angelegter nicht experi- \\
\end{tabular}

Die vorliegenden Empfehlungen wurden von einer Expertengruppe im informellen Konsens erarbeitet und nach Begutachtung durch unabhängige Gutachter in revidierter Form vom Vorstand der Deutschen Gesellschaft für Pneumologie verabschiedet.

Es muss betont werden, dass Empfehlungen häufig einen Kompromiss darstellen, wobei im Expertenkreis in den meisten Aussagen Konsens bestand. Die Empfehlungen richten sich an bronchoskopisch tätige bzw. weiterbildende und in der Weiterbildung befindliche Ärzte sowie das endoskopische Assistenzpersonal. Zusätzlich zu dieser Publikation wird eine ausführliche Fassung im Internet veröffentlicht.

\section{Anforderungen an die Strukturqualität}

\section{Persönliche Qualifikation der endoskopierenden Ärzte}

Die Voraussetzungen zum eigenständigen Durchführen von Bronchoskopien sind in der Weiterbildungsordnung Innere Medizin/Pneumologie der Landesärztekammern festgelegt.
Die Mindestanforderung für selbständig diagnostisch-bronchoskopisch tätige Ärzte beträgt 200 eigenständig durchgeführte Bronchoskopien.

Die Ausbildung sollte in folgende Schritte gegliedert sein:

- 30 Bronchoskopien „Beobachtung“ und „Übung am Phantom": Die/der Lernende wohnt der Untersuchung als Beobachter bei. Dabei werden der Ablauf der Untersuchung, die Handhabung des Bronchoskops und des diagnostischen Instrumentariums und das Management allgemeiner Schwierigkeiten erlernt, die im Rahmen der Untersuchung auftreten können. Parallel werden während dieser Zeit am Phantom manuelle Fähigkeiten im Manövrieren des Gerätes erworben und die endobronchiale Topographie studiert.

- 15 Bronchoskopien in Assistenz: Der Lernende führt nur den Rückzug des Gerätes eigenständig durch.

- 15 Bronchoskopien eigenständig und vollständig unter Supervision.

- 20 bis 40 Bronchoskopien eigenständig und vollständig unter Supervision.

Dabei werden erlernt und geübt:

- Die Durchführung von Bürstenabstrichen, bronchoalveolären Lavagen, Schleimhautbiopsien, transbronchialen $\mathrm{Na}-$ delpunktionen und transbronchialen Lungenbiopsien und

- das Management von Blutungen, Hypoxämien und Pneumothorax.

- Im Rahmen der weiteren 200 Bronchoskopien der Lernphase sollten die ersten 100 Bronchoskopien nur dann selbständig durchgeführt werden, wenn ein bronchoskopieerfahrener Arzt im Hause präsent ist.

Von jedem bronchoskopierenden Arzt muss die Teilnahme an mindestens einem Fort- bzw. Weiterbildungsseminar nachgewiesen werden. Dieses Seminar muss die von der Deutschen Gesellschaft für Pneumologie aufgeführten Mindestinhalte abdecken (siehe Punkt 6) und sollte künftig mit einer mündlichen Prüfung abgeschlossen werden. Zum Erhalt der Qualifikation muss der Nachweis von jährlich mindestens 50 eigenverantwortlich durchgeführten Bronchoskopien erbracht werden.

Als Voraussetzung für die eigenverantwortliche Durchführung interventioneller Eingriffe wird der Nachweis von mindestens 20 starren Bronchoskopien dringend empfohlen. Vor der selbständigen Durchführung interventioneller Eingriffe ist eine ganztägige Hospitation von mindestens 3 Monaten Dauer in einem entsprechend ausgewiesenen Zentrum erforderlich. Dabei sollten mindestens 10 rekanalisierende Maßnahmen in Assistenz bzw. unter Supervision in eigenständiger Verantwortung nachgewiesen werden.

\section{Qualifikation des Assistenzpersonals}

Der endoskopierende Arzt ist dafür verantwortlich, dass mitwirkendes Assistenzpersonal eine fachspezifische Qualifikation besitzt. Es hat sich gezeigt, dass die Ausbildung des Personals in externen Kursen die Effizienz und Sicherheit der praktischen Arbeit verbessert [18]. Zum Thema Qualifikation des Assistenzpersonals wurden im Oktober 1997 von der Deutschen Gesellschaft für Endoskopieassistenzpersonal (DGEA), dem Deutschen Berufsverband für Krankenpflege (DBVK) und der Deutschen Krankenhausgesellschaft (DKG) Richtlinien vereinbart [19]. Diese re- 
geln die Anerkennung des Berufsbildes der Endoskopiefachkraft und den entsprechenden Lehrplan einer zweijährigen berufsbegleitenden Ausbildung.

\section{Räumliche, apparative und hygienische Anforderungen Räumliche Anforderungen}

Die folgenden Anforderungen für Raumbedarf gründen sich im Wesentlichen auf die aktuellen Empfehlungen der Kommission für Krankenhaushygiene und Infektionsprävention beim RobertKoch-Institut [14]. Als Mindestvoraussetzungen in der Klinik sollten folgende funktionelle Bereiche bzw. Räume vorhanden sein.

- Ein Untersuchungsraum (hier kann auch die Vorbereitung des Patienten erfolgen, z. B. der intravenöse Zugang gelegt und die Lokalanästhesie verabreicht werden.)

- Ein separater Raum für die Geräteaufbereitung (mit reiner und unreiner Zone).

- Eine Warte- und Überwachungszone.

- Ab 8 Personen sollte für das Personal ein Aufenthaltsraum vorhanden sein.

Der Untersuchungsraum soll so groß bemessen sein, dass der Untersuchungstisch von allen Seiten zugänglich ist und der Patient auch aus einem Krankenbett umgelagert werden kann. Die Tür muss so breit sein, dass ein Patient ggf. im Krankenbett bzw. auf einer Liege in den Untersuchungsraum gefahren werden kann (C) [20-23]. Jeder Untersuchungsraum muss über einen hygienischen Händewaschplatz verfügen (A) [24]. Für Röntgenuntersuchungen (z.B. während der transbronchialen Lungenbiopsie) gelten besondere Anforderungen des Strahlenschutzes (C). Eine Möglichkeit zur Abdunklung des Tageslichtes muss vorhanden sein [25].

Der Aufbereitungsraum muss ausreichend groß sein und über genügend Arbeitsflächen verfügen, um eine arbeitstechnische Trennung in einen reinen und unreinen Bereich zu ermöglichen (A) [20-23]. Auch für Reinigungs- und Desinfektionsgeräte muss Platz vorhanden sein. Wegen der erhöhten Raumluftbelastung mit Desinfektionsmitteln/Dämpfen muss die Möglichkeit zur ausreichenden Lüftung gegeben sein $(A)[26,27]$. Die unreine Zone des Aufbereitungsraumes soll umfassen: ein Ausgussbecken für abgesaugtes organisches Material, Becken bzw. Wannen zum Einlegen und zur Reinigung benutzter Endoskope, ausreichende Arbeitsflächen, einen Dichtigkeitsmesser für die Endoskopaufbereitung, ein Ultraschallbad zur Aufbereitung von endoskopischem Zusatzinstrumentarium und einen hygienischen Händewaschplatz (A) [24]. Im Desinfektionsbereich müssen medizinische Druckluft und Druckluftpistolen vorhanden sein. Sofern die Sterilisation von endoskopischem Zusatzinstrumentarium nicht in einer Sterilisationsabteilung oder extern erfolgt, muss ein Sterilisator installiert sein.

Zwingend erforderlich ist die Verfügbarkeit einer Warte- und Überwachungszone, in der die Patienten vor bzw. nach der Untersuchung beobachtet/überwacht werden können. Wünschenswert ist eine optisch/akustische Trennung der beiden Bereiche. Die Aufwachzone muss mit einem Waschbecken, Anschlüssen für Sauerstoff und Absaugung, einem Pulsoximeter, einem Einkanal-EKG und einer Patientenrufanlage ausgestattet sein.
Abschließend ist darauf hinzuweisen, dass getrennte Toiletten für Patienten und Personal zur Verfügung stehen müssen. Die Personaltoilette muss über einen hygienischen Händewaschplatz verfügen $(A)[24]$.

\section{Apparative Ausstattung, Instrumentarium}

Die Ausrüstung mit Endoskopiegeräten richtet sich nach der Untersuchungszahl und dem Spektrum der jeweiligen Abteilung. Für die Durchführung der flexiblen und starren Bronchoskopie sind unterschiedliche Mindestanforderungen erforderlich. Über 90\% der diagnostischen Bronchoskopien werden derzeit mit flexiblen Glasfiberoptiken durchgeführt, die unterschiedlichen Anforderungen genügen müssen.

Zur Grundausstattung gehören deshalb je ein Bronchoskop mit einem kleineren $(<5 \mathrm{~mm})$ sowie einem größeren $(>5,6 \mathrm{~mm}) \mathrm{Au}-$ ßendurchmesser, verschiedene Typen von Zangen, Bürsten und Nadeln. Zu therapeutischen Zwecken und für den Notfall müssen verschiedene Absaugkatheter, Ballons für die Bronchusblockade und Notfalltuben vorhanden sein.

Die Verfügbarkeit eines starren Bronchoskops mit Beatmungsgerät ist in kleineren Untersuchungseinheiten nicht zwingend, sollte aber in Abteilungen mit über 500 Bronchoskopien pro Jahr gegeben sein. Unverzichtbar sind in jeder Endoskopieabteilung leistungsstarke Absaugeinheiten, eine höhenverstellbare Untersuchungsliege mit anstellbarem Kopfteil, fakultativ (je nach Spektrum) eine Durchleuchtungseinheit, Anschlüsse für die Versorgung mit Sauerstoff und Druckluft sowie je nach Arbeitsspektrum Laser, Argon-Plasma-Koagulator, Kryosonde und Elektrokauter.

Zur Beherrschung von Notfällen sind ein Notfallkoffer und ein Defibrillator, ein Pneumothoraxdrainagenset, diverse Tuben (u.a. Bronchosafe), Tamponadematerialien sowie ein Notfallkoniotomiebesteck erforderlich.

\section{Hygienische Anforderungen}

Grundlage hierfür ist ein Hygieneplan entsprechend den Empfehlungen der Kommission für Krankenhaushygiene und Infektionsprävention beim Robert-Koch-Institut (RKI) [15]. Diese gelten sowohl für die bronchoskopischen Untersuchungen im Krankenhaus wie auch in der ambulanten fachärztlichen Praxis. Jede Aufbereitung von Bronchoskopen muss von geschultem Personal in einem eigens für diesen Zweck ausgestatteten Raum (mit reiner und unreiner Zone) durchgeführt werden (C). Es sollen ausschließlich fachgerechte Reinigungs-, Desinfektions- und Sterilisationsverfahren mit geprüften und für wirksam befundenen Mitteln durchgeführt werden.

Die Aufbereitung von Endoskopen und von instrumentellem Zubehör muss unmittelbar nach dem Einsatz erfolgen, um eine Antrocknung von mikrobiell kontaminiertem Material zu vermeiden. Wesentlicher Schritt ist daher eine sorgfältige und gewissenhafte manuelle Grobreinigung bzw. Vorreinigung einschließlich der Bürstenreinigung des Instrumentierkanals (A) $[28,29]$. $\mathrm{Zu}$ dieser Vorreinigung werden Lösungen oberflächenaktiver nicht schäumender Substanzen, enzymatische Reiniger oder nachgewiesenermaßen kombiniert reinigende und desinfizierende Lösungen verwendet [29]. Aldehyde können zur Fixierung 
von Eiweiß führen, ihr Einsatz bei der Reinigung wird daher nicht empfohlen (A) [28]. Nach der Vorreinigung folgt die Desinfektion durch manuelle, halbautomatische oder automatische Verfahren. Bester Stand der Technik ist die maschinelle, chemische oder chemothermische Desinfektion [30,31]. Ihre Vorteile sind Standardisierung, hohe Sicherheit, Arbeitserleichterung, geringer Personalbedarf, geringere Umgebungsbelastung und automatische Dokumentation. Nachteile sind hohe Investitionskosten, spezielle bauliche Anforderungen, längere Aufbereitungszeit und hoher Energie- und Wasserverbrauch.

Die Eigenschaften eines „idealen Desinfektionsmittels“ sollten umfassen: Kurze Einwirkzeit, breite Wirksamkeit, fehlende Toxizität, Nebenwirkungsfreiheit und Schonung des Materials. Als Desinfektionsmittel der Wahl gilt derzeit eine $2 \%$ ige Glutaraldehydlösung [29]. Andere Desinfektionsmittel (z.B. Isopropanol $70 \%$ ig) haben Lücken im erforderlichen Wirkungsspektrum [32]. Weitere Desinfektionsmittel, z.B. Aminderivate oder Peressigsäure werden für die maschinelle Aufbereitung flexibler Endoskope zur Zeit getestet. So ist Peressigsäure gegenüber einigen atypischen Mykobakterien, die gegen Glutaraldehyd resistent sind, sehr sensibel, schädigt aber die Ummantelung der Bronchoskope. Grundsätzlich sind nur Desinfektionsmittel mit nachgewiesener antibakterieller, antiviraler und fungizider Wirksamkeit zu verwenden $(\mathrm{A})[33,34]$.

Endoskopisches Zusatzinstrumentarium, das die Mukosa penetriert (z. B. Biopsiezangen, Nadeln), muss im Rahmen der Aufbereitung sterilisiert werden (A) [29,35]. Bei Einweginstrumenten wird die Sterilität vom Hersteller garantiert. Bei Aufbereitung mehrfach verwendbaren Instrumentariums muss der Sterilisationserfolg vom Betreiber nachvollziehbar sichergestellt werden (C) $[35,36]$. Injektionsnadeln sind grundsätzlich als Einwegprodukt einzusetzen, da eine Aufbereitung von Blut in kontaminierten Injektionsnadeln technisch schwierig ist und ein hohes Verletzungs- und Infektionsrisiko birgt (A) [35]. Um Kontaminationen durch Spüllösungen zu vermeiden, sind auch die Optikspülflasche und der Anschlussschlauch arbeitstäglich aufzubereiten (A) [37].

Neben den genannten instrumentellen Maßnahmen sind folgende wichtige patienten- und personalbezogenen Regeln zu beachten:

- Die Untersuchung infektiöser Patienten erfolgt stets am Ende des Programms. Hierfür sind aber keine gesonderten Geräte zur Verfügung zu halten.

- Der Schutz des Personals wird durch konsequentes Tragen von Einmalkleidung, Einmalhandschuhen, gegebenenfalls Schutzbrille bzw. Gesichtsmaske gewährleistet. Letztere wird vor allem beim Bronchoskopieren von Patienten mit multiresistenter Tuberkulose und HIV empfohlen [38]. Es ist ein erhöhtes Infektionsrisiko bei Hepatitis B/C, HIV, Tuberkuloseund Pilzinfektionen zu beachten. Stichverletzungen mit Nadeln stellen dabei das größte Risiko dar [39]. Alle Mitglieder der Bronchoskopieabteilung sollten daher gegen Hepatitis B geimpft werden (B).

- Wo immer möglich, sollten Einmalartikel benutzt werden. Das Tragen von Gummihandschuhen ist obligat, zur Vermeidung einer Latexsensibilisierung sollten möglichst nicht gepuderte Handschuhe benutzt werden [40].
- Vor und nach jeder Endoskopie ist eine hygienische Händedesinfektion durchzuführen.

- Beim Einsatz von Aldehyden sind Schutzmaßnahmen zu beachten wie: Hautschutz, Abdeckung der Wannen und der Automaten, lüftbare Räume bzw. Absaugvorrichtung.

Nach jeder Untersuchung sind die Patientenliege und die Arbeitsfläche zu reinigen. Nach Programmende werden kontaminierte Flächen einer Wischdesinfektion unterzogen.

Um den genannten Anforderungen gerecht zu werden, soll ein angemessener Teil (z.B. 50\%) des Endoskopiepflegepersonals die Fachweiterbildung „Endoskopie“ besitzen (Empfehlung der Deutschen Krankenhausgesellschaft (DKG)) [19]. Für die Mitarbeiter der Endoskopieeinheit sind regelmäßige und fachspezifische Schulungen erforderlich, die auch dokumentiert werden müssen (C) [19]. Die innerbetrieblichen Verfahrensweisen zur Infektionshygiene müssen in Hygieneplänen festgelegt werden (C) [19]. Eine enge Zusammenarbeit mit Hygienefachpersonal (z.B. Krankenhaushygieniker, Hygienefachkraft) ist zu empfehlen (A) [19].

\section{Organisatorische Anforderungen Untersuchungszeit}

Der durchschnittliche Zeitbedarf des ärztlichen Dienstes für eine Bronchoskopie beträgt etwa 55 Minuten. Er setzt sich zusammen aus der Zeit für die Vorbereitung (Gespräch mit dem Patienten, Kontrolle der Vollständigkeit von Voruntersuchungen, Legen eines intravenösen Zugangs) (ca. 20 Minuten), der eigentlichen Untersuchung (ca. 20 Minuten) und der Nachsorge (ca. 15 Minuten). Dieser Zeitbedarf variiert in Abhängigkeit von Frequenz und Art des Eingriffs und schließt nur die unmittelbar bronchoskopiebezogene Zeitspanne ein (Tab. 2). Zur Vorbereitungszeit und Nachsorge im weiteren Sinn zählen aber auch die Prüfung der Indikationen und Risikofaktoren, die Durchsicht der Krankenakte, die Aufklärung mit Unterschrift sowie die abschließende Befundung und Dokumentation. Für den Arzt addiert sich damit der Gesamtbedarf für eine Routinebronchoskopie auf ca. 90 Minuten. Er verlängert sich bei speziellen diagnostischen und therapeutischen Maßnahmen entsprechend (Tab. 2) (B) [41].

Für eine Routinebronchoskopie sind 2 Funktionskräfte erforderlich, deren Zeitbedarf auf jeweils etwa 80 Minuten veranschlagt wird.

\section{Ärztliche Aufgaben}

Der endoskopierende Arzt hat für die gesamte Organisation mit Voruntersuchungen, Aufklärung, Nachbeobachtung, Nachbetreuung und Folgebehandlungen Sorge zu tragen. Folgende Voraussetzungen müssen erfüllt sein:

- Nach der Bronchoskopie ist der endoskopierende Arzt für den Patienten unmittelbar erreichbar. Die Betreuung kann gegebenenfalls von einem namentlich benannten, fachkompetenten Arzt übernommen werden. Die Vertretung ist zu dokumentieren.

- Der endoskopierende Arzt beteiligt sich an externen und internen Qualitätssicherungsmaßnahmen der Ärztekammern und/oder der Kassenärztlichen Vereinigungen.

- Die Aufklärung des Patienten über den geplanten Eingriff, über Risiken, Alternativen und über die Nachbehandlung 
Tab. 2 Zeitbedarf für die Bronchoskopie**

\begin{tabular}{|c|c|c|c|c|c|c|}
\hline & \multicolumn{3}{|c|}{ Ärzte } & \multicolumn{3}{|c|}{ Pflegekräfte } \\
\hline & $\boldsymbol{V}^{*}$ & $U^{*}$ & $N^{*}$ & $\boldsymbol{V}^{*}$ & $U^{*}$ & $N^{*}$ \\
\hline $\begin{array}{l}\text { 1. Routine-Bronchoskopie } \\
\text { mit einfacher Biopsie und } \\
\text { Sekretgewinnung }\end{array}$ & $20^{\prime}$ & $20^{\prime}$ & $15^{\prime}$ & $30^{\prime}$ & $3 \times 20^{\prime}$ & $\begin{array}{l}30^{\prime} \\
\left(+30^{\prime} \text { bei }\right. \\
\text { Narkose })\end{array}$ \\
\hline \multicolumn{7}{|c|}{ zusätzlicher Zeitbedarf bei besonderen Untersuchungsmethoden: } \\
\hline $\begin{array}{l}\text { - Sondieren mit Katheter } \\
\text { bzw. Bürste }\end{array}$ & & $10^{\prime}$ & & & $3 \times 10^{\prime}$ & \\
\hline $\begin{array}{l}\text { - transbronchiale Lun- } \\
\text { genbiopsie }\end{array}$ & & $10^{\prime}$ & & & $3 \times 10^{\prime}$ & \\
\hline $\begin{array}{l}\text { - transbronchiale } \\
\text { Lymphknotenbiopsie }\end{array}$ & & $10^{\prime}$ & & & $3 \times 10^{\prime}$ & \\
\hline $\begin{array}{l}\text { - bronchoalveoläre } \\
\text { Lavage }\end{array}$ & & $10^{\prime}$ & & & $3 \times 10^{\prime}$ & \\
\hline $\begin{array}{l}\text { 2. Bronchoskopie mit spe- } \\
\text { ziellem Eingriff, z. B. Tam- } \\
\text { ponade bei schwerer Blu- } \\
\text { tung, Fremdkörperentfer- } \\
\text { nung, therapeutischer } \\
\text { Lavage, Laser-Behand- } \\
\text { lung, intrabronchialer } \\
\text { Strahlentherapie }\end{array}$ & $15^{\prime}$ & $50^{\prime}$ & $20^{\prime}$ & $15^{\prime}$ & $2 \times 50^{\prime}$ & $\begin{array}{l}40^{\prime} \\
\left(+30^{\prime} \text { bei }\right. \\
\text { Narkose })\end{array}$ \\
\hline $\begin{array}{l}\text { 3. Bedside-Bronchoskopie } \\
\text { z. B. auf der Intensiv- } \\
\text { station }\end{array}$ & $10^{\prime}$ & $20^{\prime}$ & $15^{\prime}$ & $20^{\prime}$ & $20^{\prime}$ & $20^{\prime}$ \\
\hline
\end{tabular}

$\mathrm{V}^{*}=$ Vorbereitung

$\mathrm{U}^{*}=$ Untersuchung

$\mathrm{N}^{*}=$ Nachsorge

**Durchschnittswerte aus verschiedenen Krankenhäusern in Minuten

wird schriftlich dokumentiert. Dies gilt auch für die Einverständniserklärung des Patienten zum Eingriff, zur Medikation und gegebenenfalls zur Allgemeinanästhesie.

- Der Befund wird zeitgerecht erstellt und übermittelt.

- Die Kooperation zwischen vorbehandelndem, endoskopierendem und nachbehandelndem Arzt ist gewährleistet.

- Der für die Endoskopie verantwortliche Arzt trägt gegebenenfalls für eine stationäre Behandlung Sorge, die z.B. als Folge von Komplikationen erforderlich ist.

\section{Anforderungen an die Prozessqualität}

\section{Indikation}

Der endoskopierende Arzt entscheidet über Art, Umfang und Dringlichkeit der Endoskopie. Dies geschieht in Abstimmung mit dem Patienten, unter Einbeziehung der Vorinformation des überweisenden Arztes, unter Abwägung seines häuslichen Umfeldes und - erfolgt der Eingriff unter Vollnarkose - in Abstimmung mit dem Anästhesisten. Die allgemeinen Indikationen und die Indikationen für spezielle Untersuchungsmethoden sind in der Anlage aufgeführt (Anlage 1).

\section{Kontraindikationen}

Relative Kontraindikationen liegen nur bei Patienten vor, bei denen sich aus allgemeinen klinischen und/oder funktionellen Gründen aus der angestrebten bronchoskopischen Diagnosesicherung keine anderen als die bisherigen therapeutischen Konsequenzen ergeben. Bei elektiven Eingriffen werden relative
Kontraindikationen von Art und Umfang des Eingriffs bestimmt. Sie sind in Anlage 2 aufgelistet. Im Rahmen der Notfallmedizin gibt es keine absoluten Kontraindikationen. Das Risiko Herzinfarkt wird im Folgenden gesondert erläutert.

Die Bronchoskopie kann zu kardialen Ischämien führen, insbesondere bei Patienten über 60 Jahre [42]. Retrospektive Studien zeigten aber, dass bronchoskopische Untersuchungen nach Herzinfarkt sicherer waren als bisher angenommen $[43,44]$. Voraussetzung ist aber hierfür, dass eine kontinuierliche Sauerstoffgabe, ein EKG-Monitoring, und eine adäquate Sedierung erfolgen und die Untersuchung nicht während akuter Ischämie durchgeführt wird [45]. Es wird daher empfohlen, die Durchführung von Bronchoskopien innerhalb von 4 Wochen nach einem Herzinfarkt möglichst zu vermeiden (C) [46].

\section{Formale Voraussetzungen}

Der Umfang der für eine elektive Bronchoskopie erforderlichen Voruntersuchungen richtet sich nach der Grunderkrankung und den Begleiterkrankungen sowie dem Gesundheitszustand des Patienten. Zur Vermeidung von Aspirationen muss die empfohlene Nahrungskarenz zwischen fester und flüssiger Nahrung unterscheiden. Die Nahrungskarenz vor der Untersuchung mit flexiblen Bronchoskopen muss für feste Bestandteile mindestens 4 Stunden, nach Stubbs 6 Stunden [47] und nach Reed mindestens 8 Stunden betragen [48]. Die Aufnahme von Flüssigkeiten ist bis 2 Stunden vor dem Eingriff gestattet. Evidenzbasierte Daten liegen hierzu allerdings nicht vor.

Vor Bronchoskopien in Vollnarkose dürfen die Patienten mindestens 8 Stunden keine Nahrung zu sich genommen haben. Flüssigkeiten in kleinen Mengen sind bis 2 Stunden vor dem Eingriff erlaubt (B) $[49,50]$.

Das Risiko signifikanter Blutungen bei transbronchialer Lungenbiopsie liegt zwischen 1 und $5 \%$ und steigt bis auf $15 \%$ bei pulmonaler Hypertonie, bis $20 \%$ unter Beatmung, bis $25 \%$ bei immunsupprimierten Patienten und auf bis zu $45 \%$ bei urämischen Patienten [51 -53]. Die Einnahme von ASS zeigte in einer retrospektiven Studie keine Korrelation mit erhöhten Blutungskomplikationen [54]. Dies wurde kürzlich durch eine prospektive Studie bestätigt. Patienten, die bis 24 Stunden vor der Bronchoskopie inklusive TBLB Aspirin eingenommen hatten, wiesen keine signifikant höhere Blutungsrate auf als Patienten ohne ASS $(1,4 \%$ versus $1,1 \%)[55]$.

Zur Bronchoskopie sind folgende Voruntersuchungen obligat (bzw. verzichtbar).

- EKG, nicht älter als 14 Tage (z.B. Ausschluss eines akuten Herzinfarktes)

- Aktuelle Röntgenübersichtsaufnahmen in 2 Ebenen, nicht älter als 14 Tage

- Computertomographie bei Verdacht auf einen malignen Prozess bzw. vor geplanter transbronchialer/trachealer Biopsieentnahme

- Arterielle Blutgasanalyse und Spirometrie, nicht älter als 14 Tage

- Eine routinemäßige Bestimmung der Thrombozytenzahl, der Prothrombinzeit und der partiellen Thromboplastinzeit ist nur bei Patienten erforderlich, die ein erhöhtes Blutungsrisiko 
aufweisen oder bei Patienten, bei denen eine transbronchiale Lungenbiopsie geplant ist (C) [56].

- Wenn die Entnahme von Biopsien geplant ist, sollte Marcumar mindestens 3 Tage, Heparin einen Tag vor der Bronchoskopie abgesetzt werden. Gegebenenfalls muss eine VitaminK-Substitution durchgeführt werden [57]. ASS darf bis zu einer Dosis von $100 \mathrm{mg}$ täglich einen Tag vor Bronchoskopie eingenommen werden (A) [55].

- Zur Handhabung neuerer Antikoagulantien wie Ticlopidin oder Clopidogrel gibt es keine ausreichenden Daten, es ist aber zu empfehlen, diese Medikamente 7 bis 11 Tage vor einem Risikoeingriff (z. B. TBLB) abzusetzen (C) [58].

- Serumelektrolyte und Cholinesterase bei Untersuchungen in Vollnarkose.

- Die prophylaktische Gabe von Antibiotika wird empfohlen bei Patienten mit Z. n. Milzexstirpation oder zur Endokarditisprophylaxe (B) $[59,60]$.

\section{Aufklärung}

Die Aufklärung umfasst zunächst die Klärung der Voraussetzungen. Umfang und Zeitpunkt der Aufklärung hängen von der Art und Schwere des Eingriffs ab. Die Aufklärung muss schriftlich (oder vor Zeugen) durch den untersuchenden Arzt oder einen fachkompetenten Vertreter, bei Eingriffen in Vollnarkose zusätzlich durch den Anästhesisten erfolgen. Der Aufklärungstext kann durch einen handschriftlichen Zusatz ergänzt werden, z. B. sollte vor der Durchführung transbronchialer Biopsien auf die Möglichkeit eines Spätpneumothorax hingewiesen werden. Durch organisatorische Maßnahmen ist eine räumlich und zeitlich vom endoskopischen Eingriff abgesetzte Aufklärung zu erreichen. Bei stationären Patienten sollte zwischen Aufklärung und Untersuchung mindestens eine Nacht liegen. Das aufklärende Gespräch darf nicht im Untersuchungsraum und nicht nach der Prämedikation erfolgen. Ist im Notfall keine Aufklärung möglich, ist eine genaue Dokumentation umso zwingender.

Bei ambulanten Untersuchungen empfiehlt es sich, den Patienten vor der Bronchoskopie, etwa im Rahmen einer Sprechstunde einzubestellen und zeitlich vorgezogen ( $\geq 12$ Stunden) aufzuklären. Der Patient ist zu instruieren, dass er nach Sedierung oder Vollnarkose bis 24 Stunden nach dem Eingriff nicht oder nur eingeschränkt verkehrstauglich oder geschäftsfähig ist. Falls eine Sedierung verabreicht wurde, ist für den Nachhauseweg eine Begleitperson erforderlich.

\section{Prämedikation}

Es gibt große Unterschiede in der praktischen Handhabung und Durchführung der Prämedikation, zumal bislang auch international keine Richtlinien und allgemein gültigen Empfehlungen zur Prämedikation existieren [9-12]. Die Prämedikation sollte sich nach Art und Schwere des Eingriffs, Vorerkrankungen, aktuellem Gesundheitszustand, Arzneimittelunverträglichkeit und nach dem Wunsch des Patienten richten. Eine intravenöse Prämedikation ist nicht obligat [61]. Für Lokalanästhetika gilt eine Dosisobergrenze.

Die Lokalanästhesie der Nasen-Rachen- und Bronchialschleimhaut kann auf verschiedene Art erfolgen [62,63]. Die Nasenschleimhäute werden am besten mit Lidocain-Gel, der Rachen, die Stimmbänder und die oberen Trachealabschnitte mit $2 \%$ igem
Lidocain über einen Rachenzerstäuber anästhesiert. Alternativ kann Lidocain auch in vernebelter Form über einen Inhalator gegeben werden [64]. Nach Passage der Stimmritze erfolgt die Anästhesie der Bronchialschleimhäute mittels fraktionierter Bolusgabe von 1 bis $2 \%$ igem Lidocain durch den Arbeitskanal, während das Bronchoskop sukzessive vorgeschoben wird. In prospektiven Studien wurden bei Dosen von $600 \mathrm{mg}$ Lidocain keine Zeichen von Toxizität (zerebraler Krampfanfall, Herzinsuffizienz) beobachtet $[65,66]$. Eine neuere Studie empfahl eine Maximaldosis von $8,2 \mathrm{mg} / \mathrm{kg}$. Dies entspricht einer Dosis von $574 \mathrm{mg}$ bzw. 29,3 ml eines $2 \%$ igen Lidocains für einen 70 Kilo schweren Patienten [65]. Aufgrund vereinzelter Todesfälle nach nicht genauer eruierbaren Dosen, empfehlen die Hersteller die Maximaldosis von 4,5 mg/kg nicht zu überschreiten [67]. Dies entspricht für einen $70 \mathrm{~kg}$ schweren Patienten einer obersten Gesamtdosis von $300 \mathrm{mg}$ bzw. $15 \mathrm{ml}$ eines $2 \%$ igen Lidocains. Für Kinder, Patienten über 80 Jahre und Patienten mit Herz- und Leberinsuffizienz liegt die Höchstdosis bei $80 \mathrm{mg}$ (C) [67,68].

Der Stellenwert einer intravenösen Sedierung, beispielsweise mit Lorazepam, Midazolam und Phenoperidin wird in älteren Publikationen kontrovers beschrieben [69-71]. Die Ergebnisse jüngerer Studien sprechen klar für eine sedierende Prämedikation $[60,72,73]$. Zweck der Sedierung ist, die Beeinträchtigung des Patienten zu reduzieren und damit den Eingriff für beide Seiten - Patient und Untersucher - zu erleichtern [74,75]. Midazolam war in einer prospektiven randomisierten Studie der Kombination von Alfentanil-Propofol überlegen [76]. Die Akzeptanz war hoch, wenn die Sedierung so titriert wurde, dass leichter Schlaf eintrat [71]. Midazolam, ein wasserlösliches Benzodiazepin, hat eine Halbwertszeit von ca. 2 Stunden und verursacht eine retrograde Amnesie. Ein großer Teil der Patienten ist daher bereit, ggf. einer zweiten Bronchoskopie zuzustimmen [71]. Eine adäquate Sedierung und Amnesie kann mit initial 2 mg erreicht werden, die gegebenenfalls nach 2 Minuten durch je $1 \mathrm{mg} / \mathrm{min}$ bis zu einer Gesamtdosis von $5 \mathrm{mg}$ ergänzt werden (A) [75-77]. Auf eine eventuelle Atemdepression ist vor allem in der Nachbeobachtung zu achten. Gegebenenfalls kann mit Flumazenil antagonisiert werden, wobei zu berücksichtigen ist, dass die antagonisierende Wirkung kürzer wirksam ist als die Wirkung von Midazolam.

Atropin wurde bisher weitgehend routinemäßig zur Prämedikation eingesetzt $[18,78]$. Nach neueren Studien führt Atropin nicht zu einer signifikanten Verminderung der Sekretproduktion. Es kann jedoch Tachykardien und Rhythmusstörungen verursachen, die Entwicklung eines Glaukoms fördern und Mundtrockenheit hervorrufen [79-82]. Die intravenöse/intramuskuläre Gabe von Atropin ist daher vor Bronchoskopien nicht routinemäßig erforderlich (A) $[78,79,82,83]$.

\section{Monitoring}

Bei flexiblen Bronchoskopien sind als Mindestmaßnahme bzw. Mindestmonitoring erforderlich:

- Legen eines intravenösen Zugangs (auch wenn keine intravenöse Sedierung erfolgt)

- Kontinuierliche Sauerstoffinsufflation über eine Nasensonde. Die Flussrate soll mindestens $21 / \mathrm{min}$. betragen. Dabei sind die Kontraindikationen bei $\mathrm{CO}_{2}$-Retention zu beachten, gegebenenfalls ist eine $\mathrm{CO}_{2}$-Messung erforderlich. Durch die Sau- 
erstoffinsufflation soll eine Sauerstoffsättigung von mindestens $90 \%$ erreicht werden, um das Risiko signifikanter Arrhythmien zu senken (B) [84-86].

- Pulsoximetrie (B) [87].

- Die routinemäßige Ableitung eines Einkanal-EKG's ist nach der Datenlage in der Literatur nur obligat bei Patienten mit koronarer Herzerkrankung und bei Patienten, die trotz $\mathrm{O}_{2}$-Substitution eine Hypoxämie aufweisen (C) [85,88-90]. Sie wird aber von den Autoren als grundsätzlich obligat empfohlen.

Starre Bronchoskopien werden ausschließlich unter anästhesiologischer Begleitung in intravenöser Allgemeinanästhesie (TIVA) und Muskelrelaxation durchgeführt. Das essenzielle technische Monitoring umfasst Einkanal-EKG, Pulsoximetrie und nichtinvasive Blutdruckmessung. Verfügbar sein muss eine Überwachung der Muskelrelaxation (z.B. durch einen peripheren Nervenstimulator). Bei Risikopatienten sollte das Monitoring problemorientiert erweitert werden (z.B. durch invasive Blutdruckmessung). Respiratorisch grenzwertige Patienten (z. B. bei Globalinsuffizienz oder bei länger dauernden interventionellen Eingriffen) benötigen eine Überwachung der Ventilation, d.h. arterielle oder transkutane Blutgase bzw. Kapnographie. Der Anästhesist sollte die Besonderheiten der Evaluierung, der Narkoseführung und Nachsorge von Patienten mit zentraler Atemwegsobstruktion und die methodischen Komplikationsmöglichkeiten bei Interventionen kennen. Er muss mindestens ein Ventilationsverfahren (z.B. intermittierende Überdruckbeatmung, nieder- oder hochfrequente Jetventilation) und die apnoeische Oxigenierung beherrschen.

\section{Untersuchungsablauf}

Der Untersuchungsablauf beinhaltet die Inspektion von Kehlkopf, Trachea und einsehbaren Bronchien unter besonderer Beurteilung der Funktion (Stimmbandbeweglichkeit, Wandmotilität), der anatomischen Verhältnisse (anatomische Varianten, Kompression, Obturation, lokale Besonderheiten wie Tumor etc.), der Schleimhaut (Rötung, Atrophie, Drüsenausführungsgänge, Blutung etc.) und der Sekretion (Menge, Farbe, Konsistenz). Nach der Inspektion erfolgt die gezielte Materialgewinnung zur mikrobiologischen, zytologischen und histologischen Diagnostik. Gewebeentnahmen zur Diagnostik lokalisierter Prozesse außerhalb des Sichtbereichs des Endoskops erfordern eine Durchleuchtungseinrichtung zum gezielten Aufsuchen des Herdes und zur Erfassung von Komplikationen.

Die Durchleuchtungskontrolle bei transbronchialer Lungenbiopsie (TBLB) wird nicht einheitlich durchgeführt. Bei Patienten mit diffusen Lungenerkrankungen fand sich eine Pneumothoraxinzidenz von 3\% [91,92]. Fragebogenerhebungen ergaben, dass die Zahl der Pneumothoraxes signifikant reduziert wurde, wenn die TBLB unter Durchleuchtungskontrolle durchgeführt wurde [8]. Dies wurde auch in einer neueren Untersuchung von Smyth bestätigt, bezog sich aber nur auf drainagepflichtige Pneumothoraces [10]. In drei retrospektiven Studien fanden sich jedoch zwischen den Gruppen mit und ohne Durchleuchtung während TBLB keine signifikanten Unterschiede im Auftreten von Pneumothoraces [93-95]. Eine Durchleuchtungskontrolle bei TBLB ist daher bei diffusen Prozessen nicht obligat (B) [93-95]. Sie ist jedoch bei lokalisierten Prozessen aus Gründen der Zielgenauigkeit unbedingt erforderlich $[96,97]$.

Es ist ungewöhnlich, dass sich nach mehr als einer Stunde nach TBLB ein Pneumothorax entwickelt [98,99]. Die radiologische Kontrolle ergab in zwei prospektiven Einzelstudien in einer bzw. vier Stunden nach TBLB eine drainagenpflichtige Pneumothoraxrate von 0,9 bzw. 0,68\%. Sieben Stunden nach TBLB wurde kein Pneumothorax mehr beobachtet $[98,100]$. Die TBLB kann somit auch unter ambulanten Bedingungen durchgeführt werden (B). Es wird empfohlen, eine Stunde nach TBLB einen Röntgenthorax zum Ausschluss eines Pneumothorax anzufertigen (B) $[98-100]$.

Voraussetzungen für die Durchführung transbronchialer/-trachealer Nadelbiopsien (TBNA) sind:

- das Vorliegen eines aktuellen CT's und

- die Verfügbarkeit spezieller Nadeln (z.B. Wang 19-22 Gauge, Nadel nach Schießle).

Bei endoskopisch aufgetriebener Carina oder bei tumorverdächtiger trachealer Vorwölbung kann die TBNA im Einzelfall auch ohne Kenntnis des CT-Befundes erfolgen.

Die Durchleuchtungskontrolle bei TBNA ist nicht obligat [9]. Der endobronchiale Ultraschall befindet sich in Entwicklung und ist keine Routinemethode zur gezielten Punktion paratrachealer oder -bronchialer Gewebsstrukturen [101,102].

Für therapeutische Eingriffe müssen die Grenzen der flexiblen Bronchoskopie in Lokalanästhesie beachtet werden. Insbesondere bei hochgradigen Stenosen der Trachea mit Stridor oder eingeschränkten Reserven (Zustand nach Pneumonektomie) ist die starre Bronchoskopie in Vollnarkose vorzuziehen [2-4]. Bei Durchführung einer transbronchialen Nadelaspiration ist eine unmittelbare Untersuchung des Aspirates im Bronchoskopieraum (On-site-Pathologie) wünschenswert.

\section{Nachbeobachtung}

Über Zeit und Umfang der Patientennachbeobachtung nach Routine- oder interventionellem Eingriff gibt es in der Literatur keine einheitlichen Empfehlungen oder Richtlinien [103]. Sie sollte nach flexiblen mindestens eine, nach starren Bronchoskopien mindestens zwei Stunden betragen. Sie muss sich im Einzelnen nach dem Maß der Sedierung, der durchgeführten Maßnahme und dem Risikoprofil des Patienten richten. Die Sauerstoffinsufflation hängt unter anderem ab von Art und Schwere der vorausgegangenen Sedierung und sollte je nach aktueller Situation 1 Stunde über den Eingriff hinaus fortgesetzt werden [104]. Monitoring durch Pulsoximetrie und EKG sind nur bei entsprechender Risikokonstellation und nach starrer Bronchoskopie erforderlich. Der Patient sollte 2 Stunden nach Beendigung des Eingriffs keine Nahrung zu sich nehmen. Eine Röntgenthoraxaufnahme in Exspiration ist obligat nach transbronchialer Lungen- und Lymphknotenbiopsie und nach therapeutisch-interventionellen Eingriffen.

Sie erfolgt frühestens eine Stunde nach dem Eingriff, bei Symptomen früher. Eine zweite Röntgenaufnahme ist nur indiziert, 
wenn Symptome auftreten und beispielsweise der Verdacht auf einen Spätpneumothorax vorliegt (B) [98 - 100].

\section{Materialverarbeitung}

Entnommene Materialien müssen sofort und adäquat in Abstimmung mit den weiteruntersuchenden Institutionen verarbeitet werden. Der Untersuchungsauftrag muss exakt definiert, untersuchungsbestimmende Informationen müssen präzisiert und aktuell übermittelt werden. Die Verarbeitung bronchoskopisch gewonnener Untersuchungsmaterialien und ihr Versand erfolgt nach den Empfehlungen in Anlage 3.

\section{Ergebnisqualität, Komplikationen}

Ziel der Ergebnisanalyse ist die Möglichkeit der vergleichenden Überprüfung. Diese bezieht sich sowohl auf klinische Inhalte (wie z.B. Indikation und Komplikationen) als auch äußere Voraussetzungen (z.B. ambulante oder stationäre Durchführung). Die Ergebnisqualität bezieht sich auch auf die Erfassung von Parametern wie Materialgüte und -ausbeute, auf Diagnose/Diagnoseraten (ICD-Schlüssel), die Erfassung von Komplikationen und die Erfassung der Zufriedenheit von Patient und Einweiser. Bei der therapeutischen Endoskopie mangelt es allgemein an anerkannten objektiven Parametern wie der Erfassung von Messgrößen für die Lumenerweiterung, der Quantifizierung von Blutungen und Sekretmengen sowie der Graduierung von Symptomen. Auch eine allgemein gültige Graduierung des Schweregrades von Komplikationen liegt in der Literatur nicht vor und muss deshalb subjektiv erfolgen. Die wichtigsten bronchoskopiebezogenen Komplikationen zeigt Tab. 3. Ihre Erfassung und Quantifizierung (zumindest in leicht, mittel, schwer) sowie ihre zeitliche Zuordnung sind zu dokumentieren.

Die diagnostischen und therapeutischen Ergebnisse der Bronchoskopie müssen regelmäßig (jährlich) in internen Prüfungen erfasst werden. Für die Zukunft sind anonyme und überregional vergleichende Auswertungen in jährlichen Abständen zu empfehlen.

\section{Dokumentation}

Zielsetzung der Dokumentation ist die Vereinheitlichung und Vergleichbarkeit der Befundung. Zu dokumentieren sind die in Tab. 4 angeführten Daten. Zur Dokumentation sollte ein einheitlicher Befundbogen mit Skizze verwendet werden, in der die makroskopischen Veränderungen grafisch festgehalten und die Stellen der Biopsien markiert werden (Abb. 1). Eine Foto- oder Videodokumentation pathologischer Befunde ist dringend zu empfehlen.

Wünschenswert ist auch, dass die Daten computergestützt erfasst und verarbeitet werden. Die Vorteile dieses Vorgehens sind für die Gastroenterologie belegt (A) [105-107]. In einem klinikumfassenden Dokumentationssystem können die Ergebnisse der Materialaufarbeitung mit den weiteren diagnostischen und therapeutischen Maßnahmen und dem Verlauf verknüpft werden. Sie müssen daher strukturiert archiviert werden, um bei Bedarf rasch zur Verfügung zu stehen. Auch die Dokumenta-
Tab. 3 Komplikationen

- pulmonale Komplikationen: Pneumothorax, Blutung*, Pneumonie, Hypoxie Lungenversagen/Beatmungsnotwendigkeit, obstruktive Ventilationsstörung

- extrapulmonale Komplikationen: Arrhythmie

- Komplikationen während oder nach Interventionen

- Narkosezwischenfälle, Ventilationsprobleme, Cholinesterasemangel (längere Beatmungszeiten)

- Folgeeingriffe

- Spätkomplikationen (z. B. Heiserkeit, Halsschmerzen, Hustenreiz, Fieber)

*Blutungsscore: 0 = keine Blutung, I = keine Maßnahmen, II = Absaugen, III = Instillation von kalter $\mathrm{NaCl}$-Lösung, Tamponade durch Tupfer, $\mathrm{IV}=$ Chirurgie, $\mathrm{V}=$ Todesfall

Tab. 4 Dokumentation

\section{Stammdaten/Untersuchung}

- Patientenidentifikation (Vorname, Nachname, Geburtsdatum, Versicherung, Adresse, Hausarzt)

- Untersuchungsdatum

- Indikation, Fragestellung

- Dringlichkeit der Untersuchung (elektiv, dringlich, Notfall)

- untersuchungsrelevante Befunde (Laborparameter, Lungenfunktion, Blutgasanalyse, Röntgenbefunde, andere Voruntersuchungen)

- Aufklärungsgespräch

Befund

- Untersucher

- Assistenzpersonal

- Anästhesist

- Art der Untersuchung (diagnostisch, therapeutisch, interventionell)

- Art und Typ der verwendeten Instrumente

- Art der Intervention (Nd-YAG-Laser, Argon-Beamer, Kryosonde, etc.)

- Beginn und Ende der Untersuchung, Beginn und Ende der Überwachung

- Untersuchungsverlauf (Blutdruck, Sauerstoffsättigung, Herzfrequenz)

- Pflegedokumentation (Übergabegespräch)

- Medikation (prä-, intra-, postoperativ)

- Biopsiestellen, -art, Folgeuntersuchung

- Materialgewinnung, -versand, -diagnostik

- Bilddokumentation, Videodokumentation

- zusätzliche Daten (Durchleuchtungszeit, Laserdaten, Stentdaten)

- Befundskizze

- Befundtext

- Komplikationen

- Dokumentation der Hygienemaßnahmen

Leistungserfassung/Abrechnung

- Erfassung endoskopischer Einzelleistungen (GOÄ, EBM, DRGs)

- Diagnoseverschlüsselung (ICD 10/DRG)

Bestellwesen/Administration

- Geräteverbrauch

- Materialverbrauch

- Terminplanung

- Personaleinsatz

tion aller pflegerischen und ärztlichen Leistungen muss in einem strukturierten Befundtext erfolgen. Der Zugriff auf die Daten muss sowohl patienten- wie auch untersuchungsbezogen möglich sein. 


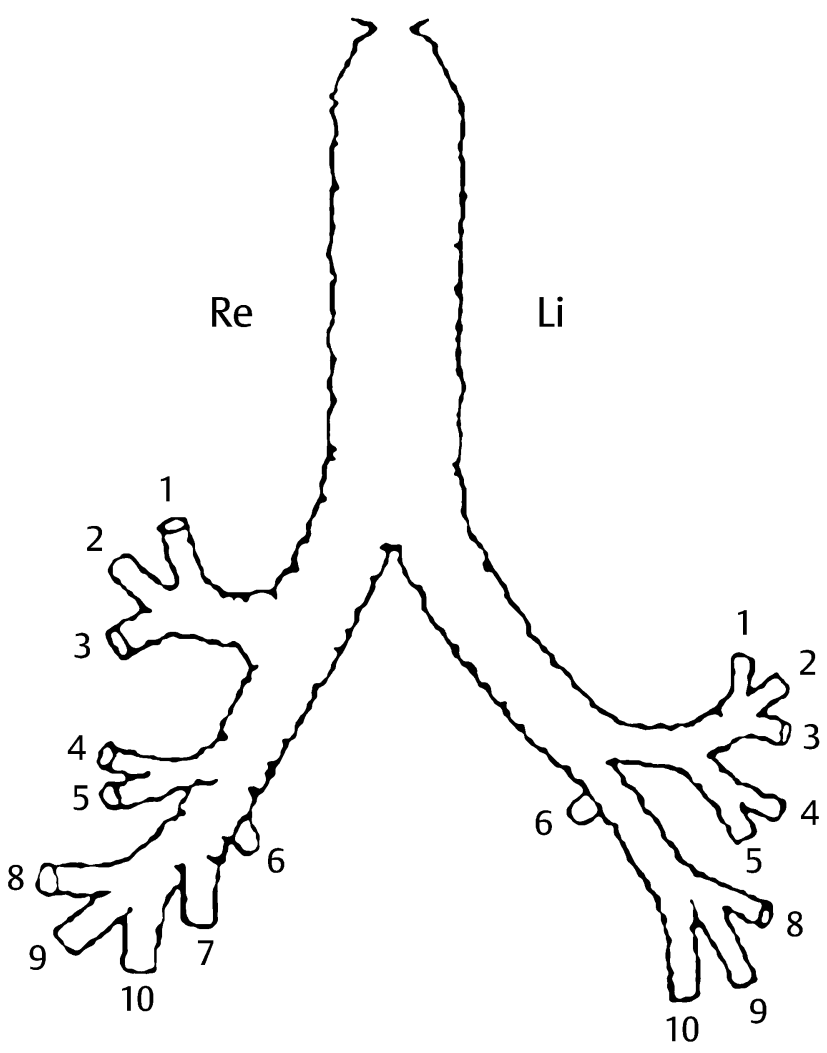

Abb. 1 Befundskizze Bronchialbaum.

Unmittelbar nach der Untersuchung erfolgt die Übermittlung des aktuellen Befundes an den weiterbehandelnden Arzt in der Klinik. Bei ambulanter Untersuchung wird dem Patienten ein Sofortbefund ausgehändigt. Nach Erhalt der Labor- und Pathologieergebnisse wird unverzüglich ein strukturierter Befundbericht an den Überweiser gesandt, der auch ein Duplikat des Pathologie- und Laborberichtes enthält.

\section{Fort- und Weiterbildung}

Ziel der Fort- und Weiterbildung ist die Vermittlung von Kenntnissen zur Durchführung und zum strukturierten Ablauf der Bronchoskopie. Allgemein verbindliche Richtlinien, wie ein solches Kurssystem, ein systematisches Lernprogramm bzw. eine strukturierte Weiterbildung gestaltet sein sollten, gibt es bisher nicht [108]. Das Programm muss in Abschnitte gegliedert sein, so dass der Lernende die Möglichkeit hat, stufenweise seine Erkenntnisse und Fähigkeiten auf dem Gebiet der Bronchologie zu erweitern [109]. Die folgenden Empfehlungen stützen sich auf etablierte Kursinhalte großer pneumologischer Ausbildungszentren, die sich in der Praxis bewährt haben und deren Lernziele auf der Basis von zertifizierten Abschlussprüfungen entwickelt wurden.

Neben der Vermittlung des bronchoskopischen Grundwissens, das nicht ohne Bezüge zur Fachpneumologie möglich ist, sollte jedes Kurssystem seine Lernziele an den speziellen Bedürfnissen der Kursteilnehmer ausrichten. Es lassen sich 3 Gruppen von Ärzten unterscheiden, auf die die Kursinhalte speziell zugeschnitten sein sollten:
Tab. 5 Lerninhalte des „Basisbronchoskopiekurses“

1. Vergleich der Indikationen und Möglichkeiten von flexibler vs. starrer Bronchoskopie

2. formale und juristische Voraussetzungen für die Bronchoskopie

3. Aufbau, Struktur und Ausstattung einer Bronchoskopieeinheit

4. Vorbereitung des Patienten

5. Anatomie des Atemwegstraktes

6. repräsentative Auswahl anatomischer Varianten des Atemwegssystems

7. Handhabung des Bronchoskops

8. Spektrum und Handhabung bronchoskopischer Hilfsmittel (z. B. Zangen, Nadeln, Bürsten, usw.)

9. Techniken zur Gewinnung, Verarbeitung und zum Versand zytologischer histologischer und mikrobiologischer Proben

10. Anforderungen und Möglichkeiten der Mikrobiologie/Virologie bei bronchoskopisch gewonnenem Untersuchungsmaterial

11. Technik der bronchoalveolären Lavage (BAL)

12. Hygieneanforderungen und Durchführung der Hygienemaßnahmen

13. Demonstration wichtiger pathologischer Befunde

14. Möglichkeiten und Indikationen der interventionellen Bronchoskopie

15. Möglichkeiten und Indikationen des endoskopischen Ultraschalls

16. bronchoskopische Notfälle

17. Notfallmanagement - Logistik, Ausstattung

18. Möglichkeiten der Sedierung und Anästhesie

19. Einsatzbereich der Bronchoskopie in der Intensivmedizin und Anästhesie

20. bronchoskopisch geführte Punktionstracheotomie (Therapie bzw. Übung am Modell)

21. Befunddokumentation

22. Struktur der Qualitätskontrolle

23. praktische Übungen am Phantom (mindestens 5 Stunden à 45 Minuten)

1. Intensivmediziner und Anästhesisten

2. Internisten

3. Pneumologen, Thoraxchirurgen, HNO-Ärzte und Pädiater.

Während die Gruppen 1 und 2 vorwiegend an der diagnostischen Fiberbronchoskopie interessiert sind, sollten die Kursinhalte der Gruppe 3 auch Kenntnisse in starrer Bronchoskopie und interventionellen Verfahren vermitteln.

Die „Basisbronchoskopiekurse“ sollten die in Tab. $\mathbf{5}$ aufgeführten Lerninhalte umfassen. Es empfiehlt sich dabei ein stufenweises Vorgehen:

1. Erlernen der Anatomie des Tracheobronchialsystems und der topographischen Beziehungen zu den großen Gefäßen des Thoraxraums. Wissensvermittlung durch Vorträge, Bild/Textmaterial und Lernen am Modell.

2. Erlernen von Aufbau und Funktion des Fiberbronchokops. Besonderes Gewicht sollte hierbei auf den richtigen Umgang mit dem Instrument sowie dessen Reinigung, Pflege, Desinfektion und Aufbewahrung gelegt werden. Handhabung und Steuerung des Fiberbronchoskops lassen sich gut an Phantomen einüben.

3. Vermittlung der Kenntnis normaler und typischer pathologischer Befunde, unterstützt durch umfangreiches Bild- und Videomaterial zur Veranschaulichung auch dynamischer Prozesse. 
Auf diesem Feld dürfte künftig die computergestützte Bronchoskopiesimulation ein virtuelles Bronchoskopieren ermöglichen, mit dessen Hilfe die einzelnen bioptischen Verfahren interaktiv erlernt und eingeübt werden können.

Begleitend zu den präklinischen Stufen ist es erforderlich, dem Anfänger durch Seminarvorträge ein fundiertes Wissen über Indikationen, Kontraindikationen und Komplikationen der Bronchoskopie zu vermitteln sowie die Wirkungsweise der zur Prämedikation und Lokalanästhesie verwendeten Medikamente zu erläutern. Erst nach Vermittlung dieser theoretischen Kursinhalte sollte die klinische Ausbildung am Patienten beginnen und schrittweise nach den unter Punkt 2.1 angegebenen Stufen praktische Sicherheit erworben werden.

Therapeutisch-interventionelle Verfahren lassen sich nur bedingt durch Weiterbildungskurse vermitteln. Tiermodelle können zwar von Vorteil sein, finden jedoch aus ethischen Gründen in der Bundesrepublik kaum Einsatz. Als Möglichkeit bietet sich ein biologisches Lungenpräparat in Form des „Erlanger Endotrainers" an, der an den bronchoskopischen Einsatz adaptiert wurde [110]. Da eine praxisbezogene Weiterbildung unverzichtbar ist, bleibt die mehrmonatige Hospitation in einem entsprechenden Zentrum für die Ausübung der interventionellen Bronchologie eine Grundvoraussetzung.

Obwohl das Ausbildungsangebot für die Bronchoskopie in der Bundesrepublik umfassend ist, muss ein von der Deutschen Gesellschaft für Pneumologie definierter Katalog von Lernzielen erstellt werden, der eine bundesweite und vergleichbare Abschlusszertifizierung erlaubt.

\section{Anlage 1}

\section{Allgemeine Indikationen \\ Klinisch}

- Aspiration, Asphyxie

- Hämoptysen

- Therapieresistenter Husten

- Rezidivierende Bronchitis, chronische Bronchitis (Beurteilung der aktuellen Therapiebedürftigkeit)

- Pneumonien, insbesondere bei Immunsuppression

- Tumorzellen oder tumorverdächtige Zellen im Sputum

- Stenoseatmung, Z. n. Tracheotomie, Beatmungsfolgen

- Dyspnoe unklarer Ätiologie

- Z.n. Thoraxtrauma mit bleibenden Verschattungen oder klinischen Beschwerden

- Thorakale Strumen

- Maligne Lymphome, Thymome, andere Mediastinaltumoren

- Verdacht auf Tuberkulose ohne Nachweis säurefester Stäbchen im Sputum

- Bronchiektasen, „angeborene“ Fehlbildungen des Tracheobronchialbaumes

\section{Radiologisch}

- Atelektasen

- Rundherde, Herde $>3 \mathrm{~cm}$ Durchmesser

- Hilusveränderungen
- Segmentale, lobäre oder ganzseitige Infiltrationen und Verschattungen

- Disseminierte Lungenveränderungen

- Lokalisierte oder einseitige Emphyseme („helle Lunge“)

- Zwerchfellhochstand

- Mediastinalverdrängung und -verziehung

- Pleuraerguss

\section{Therapeutisch}

- Bronchialtoilette

- Therapeutische Lavage

- Tumorabtragungen - konventionell, Nd-YAG-Laser, ArgonBeamer

- Photodynamische Therapie

- Lokale Strahlentherapie (Afterloading)

- Stentimplantation

- Fremdkörperextraktion (bei organischem Material mit therapeutischer Lavage)

- Lebensbedrohliche Blutungen im Tracheobronchialtrakt

- Instillation von Medikamenten

- Therapierefraktäres Asthma bronchiale

Indikationen für spezielle Untersuchungsmethoden Bürstenbiopsie, Katheterabsaugung

- Gewinnung von Material zu zytologischen, bakteriologischen und mykologischen Untersuchungen bei intrapulmonal gelegenen Lungenprozessen - Tumor, Abszess, Pneumonie, Tuberkulose

\section{Biopsie im einsehbaren Bereich}

- Schleimhautentzündung

- Schleimhautkarzinose

- Gewebeentnahme zur histologischen Tumorsicherung

- Sarkoidose, Tuberkulose

\section{Transtracheale und transbronchiale Punktionen}

- Gewinnung zytologischen und histologischen Materials aus den paratrachealen, bifurkalen, hilären und lobären Lymphknoten

- Tumorpunktionen bei sichtbarer Einengung von Trachea, Haupt- und Lappenbronchien

- Punktion von Mediastinalzysten

\section{Transbronchiale Herdpunktion}

- Punktion intrapulmonal gelegener Tumoren

\section{Transbronchiale Lungenbiopsie}

- Gewinnung von Lungengewebe zur histologischen Aufarbeitung bei disseminierten Lungenerkrankungen, Pneumonie, Tuberkulose, Tumor, etc.

\section{Bronchoalveoläre Lavage (diagnostisch)}

- Diffuse Lungenparenchymerkrankungen, Alveolarproteinose

- Infektionen (lokalisiert, z.B. Tuberkulose; generalisiert, z.B. Pneumocystis carinii)

- Asthma bronchiale

- Bronchoalveoläres Karzinom

- Karzinose 


\section{Bronchographie}

- Bronchiektasen (Klärung der Operabilität)

- Fehlbildungen

- Pleuraempyem vor Operationen

- vor rekanalisierenden Maßnahmen bei Bronchusverschlüssen

\section{Anlage 2}

\section{Relative Kontraindikationen}

Bronchoskopie in Lokalanästhesie

- Nachgewiesene Allergie gegenüber Lokalanästhetikum

- Kardiale Dekompensation

- Instabile chronisch-ischämische Herzkrankheit

- Herzrhythmusstörungen ( $\geq$ Lown IVb)

- Hirndrucksymptomatik

- Nicht kooperativer Patient

\section{Bronchoskopie in Allgemeinnarkose}

- wie bei Bronchoskopie in Lokalanästhesie

- Myasthenia gravis

- Dekompensierte Hyperthyreose

\section{Kontraindikationen zu speziellen Untersuchungsmethoden} Bürstenbiopsie, Katheterabsaugung

- V.a. arteriovenöse Fistel oder Aneurysma der Lungengefäße

- Hämorrhagische Diathese

\section{Transbronchiale Lungenbiopsie}

- Hämorrhagische Diathese

- Höhergradiges Lungenemphysem

- Pulmonale Hypertonie (Mitteldruck in der A. pulm. in Ruhe über $60 \mathrm{~mm} \mathrm{Hg}$ )

- Beatmung (PEEP)

\section{Lavage}

- Maximal $200 \mathrm{ml}$ Instillationsflüssigkeit. Bei drohender kardialer/pulmonaler Dekompensation Reduktion des Volumens auf $50-100 \mathrm{ml}$

\section{Anlage 3}

\section{Verarbeitung bronchoskopischer Untersuchungsmaterialien vor Ort und Versand Information für den Weiterbearbeiter \\ - Patientendaten (Name, Vorname, Geburtsdatum) \\ - Station/Einsender \\ - Diagnose/Verdachtsdiagnose \\ - Befund bildgebender Verfahren \\ - Angaben über Vorbefunde \\ - Erfolgte Therapie (Operation, Zytostatika, Bestrahlung, Laser) \\ - Materialbeschreibung, Kennzeichnung}

\section{Materialverarbeitung}

Biopsiematerialien

- mit sanftem Druck in eine Richtung ausstreichen
- evtl. Nadel mit physiologischer $\mathrm{NaCl}$ ausspülen

- Zylinder abrollen

\section{Imprint}

- Blut abtupfen

- Objektträger über Schnittfläche ziehen

- evtl. mit Objektträger Schnittfläche „abkratzen“

- Abstriche anfertigen bevor das entnommene Gewebe in Formalin eingelegt wird

\section{Erguss, Zystenflüssigkeit}

- $1: 9$ mit Citrat versetzen

- Immer zwei Präparate lufttrocknen, ein Präparat fixieren (z.B. Mercofix-Spray)

\section{Lagerung}

- Ausstrich für Routinefärbungen bei Raumtemperatur einige Monate

- Ausstriche für Immunzytochemie bei Raumtemperatur einige Tage

- Ausstriche für Immunzytochemie bei - $20^{\circ}$ einige Monate

- Flüssigkeiten bei $+4^{\circ}$ (evtl. Raumtemperatur) maximal 24 (48) Stunden

\section{Versand von Flïssigkeiten}

- evtl. Konservierungsmedien verwenden (Cyto Chex)

- Versand über das Wochenende vermeiden

- Plastikröhrchen verwenden

- Bruchsichere Verpackung (auch für Objektträger)

\section{Literatur}

${ }^{1}$ Deutsche Gesellschaft für Pneumologie. Empfehlungen zur diagnostischen bronchoalveolären Lavage. Pneumologie 1994; 48: 311 -323

${ }^{2}$ Deutsche Gesellschaft für Pneumologie, Arbeitsgruppe „Qualitätssicherung in der Bronchologie“. Empfehlungen zur bronchoskopischen Behandlung tracheobronchialer Verschlüsse, Stenosen und muraler maligner Tumoren. Pneumologie 1998; 52: 243-248

${ }^{3}$ Section on Respiratory Endoscopy of the German Society of Pulmonology. Recommendations for bronchoscopic treatment of tracheobronchial occlusions, stenoses and mural malignant tumours. J Bronchol 2000; 7: 133-138

${ }^{4}$ Bolliger CT, Mathur PN, Beamis JF et al. ERS/ATS statement on interventional pulmonology. Eur Respir J 2002; 19: 356 - 373

${ }^{5}$ Ernst A, Silvestri GA, Johnstone D. Interventional pulmonary procedures: Guidelines from the American College of Chest Physicians. Chest 2003; 123: $1693-1717$

${ }^{6}$ Markus A, Häußinger K, Kohlhäufl M et al. Bronchoskopie in Deutschland: Querschnitterhebung an 681 Institutionen. Pneumologie 2000; 54: 499-507

${ }^{7}$ Simpson FG, Arnold AG, Purvis A et al. Postal survey of bronchoscopic practice by physicians in the United Kingdom. Thorax 1986; 41 : $311-317$

${ }^{8}$ Prakash UBS, Offord KP, Stubbs SE. Bronchoscopy in North America: the ACCP survey. Chest 1991; 100: 1668-1675

${ }^{9}$ Colt HG, Prakash UBS, Offord KP. Bronchoscopy in North America. Survey by the American Association for Bronchology 1999. J Bronchol 2000; 7: 8-25

${ }^{10}$ Smyth CM, Stead RJ. Survey of flexible fibreoptic bronchoscopy in the United Kingdom. Eur Respir J 2002; 19: 458 - 463

11 ATS Board of Directors. Guidelines for bronchoscopy in adults. Am Rev Respir Dis 1987; 136: 1066

12 British Thoracic Society. British Thoracic Society guidelines on diagnostic flexible bronchoscopy. Thorax 2001; 56: il-i2l 
13 Wood-Baker R, Burdon J, McGregor A et al. Thoracic Society of Australia and New Zealand. Fibre-optic bronchoscopy in adults: a position paper of the Thoracic Society of Australia and New Zealand. Intern Med J 2001; 31: 479-487

${ }^{14}$ Kommission für Krankenhaushygiene und Infektionsprävention beim Robert-Koch-Institut. Anforderungen der Hygiene an die baulich-funktionelle Gestaltung und apparative Ausstattung von Endoskopieeinheiten. Bundesgesundheitsbl 2002; 45: 412 - 414

15 Kommission für Krankenhaushygiene und Infektionsprävention beim Robert-Koch-Institut (RKI). Anforderungen der Hygiene bei der Aufbereitung flexibler Endoskopie und endoskopischen Zusatzinstrumentariums. Bundesgesundheitsbl 2002; 45: 395-411

${ }^{16}$ Arbeitsgemeinschaft Wissenschaftlich-Medizinischer-Fachgesellschaften. www.uni-duesseldorf.de/WWW/AWMF/awmfleit.htm,

17 Zentralstelle der Deutschen Ärzteschaft zur Qualitätssicherung in der Medizin (AZQ). www.awzg.de,

${ }^{18}$ Honeybourne D, Neuman C. An audit of bronchoscopy practice in the United Kingdom: a survey of adherence to national guidelines. Tho$\operatorname{rax} 1997$; 52: $709-713$

19 Zentrale Arbeitsgruppe Endoskopie im Deutschen Berufsverband für Pflegeberufe. Endoskopie - ein Leitfaden für Pflegeberufe, inklusive Arbeitshilfe Endoskopie-Vorschläge zur Standardentwicklung. Eschborn: Deutscher Berufsverband für Pflegeberufe, 1996

${ }^{20}$ Euler K. Bauliche und instrumentelle Voraussetzung für die Endoskopie. Endo Praxis 1996; 3: $22-25$

21 The American Institute of Architects. Guidelines for design and construction of hospitals and health care facilities 1996-1997. Washington, D.C.: The American Institute of Architects Press, 1998

${ }^{22}$ Hohner R, Phillip J. Planung und Anforderungen - Sicht des Arztes und des Pflegepersonals. In: Phillip J, Allescher HD, Hohner R (Hrsg) Endoskopie. Struktur und Ökonomie - Planung, Einrichtung und Organisation einer Endoskopieeinheit. Bad Homburg, Englewood N.J.: Normed, International Medical Publishers, 1998: 25-30

${ }^{23}$ Mack P. Planung und Anforderungen - Sicht des Architekten. In: Phillip J, Allescher HD, Hohner R (Hrsg) Endoskopie. Struktur und Ökonomie - Planung, Einrichtung und Organisation einer Endoskopieeinheit. Bad Homburg, Englewood N.J.: Normed, International Medical Publishers, 1998: 31 - 38

${ }^{24}$ Empfehlungen des Robert Koch-Instituts. Händehygiene. Bundesgesundheitsbl Gesundheitsforsch Gesundheitsschutz 2000; 43: 230- 233

25 Becker HD, Messerschmidt E. Endoskopie in der Pneumologie. In: Philipp J, Allescher HD, Hohner R (Hrsg): Endoskopie: Struktur und Ökonomie. Bad Homburg, Englewood N.J.: Normed Verlag, 1998: $137-147$

${ }^{26}$ Alvarado CJ, Reichelderfer M. APIC guideline for infection prevention and control in flexible endoscopy. Am J Infect Control 2000; 28: $138-155$

27 Systchenko R, Marchetti B, Canard JN et al. Guidelines of the French Society of Digestive Endoscopy: recommendations for setting up cleaning and disinfection procedures in gastrointestinal endoscopy. Endoscopy 2000; 32: $807-818$

${ }^{28}$ Exner M, Leiss O, Tuschewitzki GJ. Hygiene in der Endoskopie. Z Gastroenterol 1990; 28: 635-643

${ }^{29}$ Chu NS, McAlister D, Antonopolos A. Natural bioburden levels detected on flexible gastrointestinal endoscopes after clinical use and manual cleaning. Gastrointest Endosc 1998; 48: 137-142

${ }^{30}$ Schulze L. Aufbereitung flexibler Endoskope. Praktische Erfahrungen mit verschiedenen Endoskop-Reinigungs- und Desinfektions-Automaten (ERDA) Krhs Hyg Jnferh 1997; 19: 76-82

31 Ostrowsky B. Endoscopes - current practices and controversies in infection control. Semin Infect Control 2001; 1: 267-279

32 Cowen AE. Infection and endoscopy: Who infects whom? Scand J Gastroenterol 1992; 27 (Suppl 192): 91 -96

${ }^{3}$ DGHM. Desinfektionsmittelliste der Deutschen Gesellschaft für Hygiene und Mikrobiologie (Stand 1.3. 2000). Wiesbaden: mhp, 2000

${ }^{34}$ Robert-Koch-Institut. Liste der vom Robert-Koch-Institut geprüften und anerkannten Desinfektionsmittel und -verfahren (Stand 15. 6. 1997). Bundesgesundheitsbl 1997; 40: 344-361

${ }^{35}$ Kommission für Krankenhaushygiene und Infektionsprävention beim Robert Koch-Institut. Anforderungen an die Hygiene bei der Aufbereitung von Medizinprodukten. Bundesgesundheitsbl 2001; 44: $1115-1126$
${ }^{36}$ Kozarek RA, Raltz SL, Ball TJ et al. One-year prospective study reusing disposable sphincterotomes for diagnostic and therapeutic ERCP. Gastrointest Endosc 1999; 49: 39-42

${ }^{37}$ Bader L. Ergebnisse der HYGEA-Studie. Hamburg: Jahrestagung der DGVS, 13.-16.9. 2000

${ }^{38}$ British Thoracic Society Joint Tuberculosis Committee. Chemotherapy and management of tuberculosis in the United Kingdom. Thorax 1998; $53: 536-548$

${ }^{39}$ Hanson PJ. AIDS: practising safe endoscopy. Clin Gastroenterol 1990; 4: $477-494$

40 Brehler R, Kolling R, Webb M et al. Glove powder: a risk factor for the development of latex allergy? Eur J Surg Suppl 1997; 579: 23 - 25

41 Dierkesmann R. Empfehlungen zum Personal- und Zeitbedarf bei pneumologischen Untersuchungen. Pneumologie 1994; 48: 305 - 310

42 Davies L, Misse R, Spence PMA et al. Cardiovascular consequences of fiberoptic bronchoscopy. Eur Respir J 1997; 10: 695-698

${ }^{43}$ Dweik R, Metha A, Meeker D et al. Analysis of the safety of bronchoscopy after recent acute myocardal infarction. Chest 1996; 110: $825-828$

44 Dunaga BP, Burke HL, Aquino SL et al. Fiberoptic bronchoscopy in coronary care unit patients. Chest 1998; 114: 1660-1667

45 Moisan T, Chandrasekhar A, Moran J. Arrhythmias in patients with heart disease during fiberoptic bronchoscopy. Illinois Med J 1979; 7: $27-30$

${ }^{46}$ American College of Cardiology and the American Heart Association Taskforce. Guideline update for perioperative cardiovascular evaluation for noncardia surgery, 2002. http://www.acc.org/clinicalguidelines/perio/dir/index.htm

47 Stubbs SE, McDougall JC. Preparation of the patient for bronchoscopy. In: Prakash UBS (Hrsg): Bronchoscopy. New York, 1994

48 Reed AP. Preparation of the patient for awake flexible fiberoptic bronchoscopy. Chest 1992; 101: 244-253

49 Shevde K, Trivedi N. Effects of clear liquids on gastric volume and pitt in healthy volunteers. Anesth Analg 1991; 72: $528-531$

${ }^{50}$ Kallar SK, Evertt LL. Potential risks and preventive measures for pulmonary aspiration: New concepts in preoperative fasting guidelines. Anesth Analg 1993; 77: 171 - 182

51 Blasco LH, Hernandez IMS, Garrido VV et al. Safety of transbronchial biopsy in outpatients. Chest 1991; 99: 562-565

52 Shure D. Transbronchial biopsy and needle aspiration. Chest 1989; 95: $1130-1138$

${ }^{53}$ Mitchell DM, Emerson CJ, Collins JV et al. Transbronchial lung biopsy with the fiberoptic bronchoscope: analysis of results in 433 patients. Br J Dis Chest 1981; 75: 258-262

54 Diette GB, Wiener CM, White P. The higher risk of bleeding in lung transplant recipients from bronchoscopy is independent of traditional bleeding risks. Results of a prospective cohort study. Chest 1999; 115: $397-402$

55 Herth FJ, Becker HD, Ernst A. Aspirin does not increase bleeding complication after transbronchial biopsy. Chest 2002; 122: $1461-1464$

56 Kozak EA, Brath LK. Do "screening” coagulation testpredict bleeding in patients undergoing fiberoptic bronchoscopy with biopsy? Chest 1994; 106: $703-705$

${ }^{57}$ British Committee for Standards in Haematology. Guidelines on oral anticoagulation: third edition. Br J Haematol 1998; 101: 374 - 378

${ }^{58}$ Hittelet A, Deviere J. Management of anticoagulants before and after endoscopy. Can J Gastroenterol 2003; 17: 329-332

${ }^{59}$ Dajani A, Bisno A, Chung KD et al. Prevention of bacterial endocarditis: recommendations by the American Heart Association. JAMA 1990; 264: 2919-2922

60 Simmonds NA. Recommendations for endocarditis prophylaxis. The Endocarditis Working Party for Antimicrobial Chemotherapy. J Antimicrob Chemother 1993; 31: 437-438

${ }^{61}$ Hatton MQ, Allen MB, Vathenen AS et al. Does sedation help in fiberoptic bronchoscopy? BMJ 1994; 309: 1206-1207

62 Sanderson DR. Lignocaine for topical anesthesia in fiberoptic bronchoscopy. Respiration 2000; 67: 9-10

63 Graham PR, Hay JG, Clague J et al. Comparison of three different methods used to achieve local anaesthesia for fiberoptic bronchoscopy. Chest 1992; 102: $704-707$

${ }^{64}$ Berger R, McConnell JW, Phillips B et al. Safety and efficacy of using high-dose topical and nebulized anaesthesia to obtain endobronchial cultures. Chest 1989; 95: 299-303 
${ }^{65}$ Langmarck EL, Martin RJ, Pak J et al. Serum lidocaine concentrations in asthmatics undergoing research bronchoscopy. Chest 2000; 117: $1055-1060$

${ }^{66}$ Loukides S, Katsoulis K, Tsarpalis K et al. Serum concentrations of lignocaine before, during and after fiberoptic bronchoscopy. Respiration 2000; 67: 13 - 17

${ }^{67}$ Roxane Laboratories Inc. Lidocain HC topical solution USP 4\% (package insert). Columbus, OH: Roxane Laboratories Inc, October 1996

${ }^{68}$ Day RO, Chalmers DR, Williams KM et al. The death of a healthy volunteer in a human research project: Implications for Australian clinical research. Med J Austr 1998; 168: 449-451

${ }^{69}$ Schelley MP, Wilson P, Norman J. Sedation for fiberoptic bronchoscopy. Thorax 1989; 44: 769-775

${ }^{70}$ Colt HG, Morris JF. Fiberoptic bronchoscopy without premedication. Chest 1990; 98: 1327-1330

${ }^{71}$ Williams TJ, Nicoulet J, Coleman E et al. Safety and patient acceptability of intravenous midazolam for fiberoptic bronchoscopy. Respir Med 1994; 88: $305-307$

${ }^{72}$ Maltais F, Laberge F, Laviolette M. A randomized, double-blind, placebo-controlled study of lorazepam as premedication for bronchoscopy. Chest 1996; 109: 1195-1198

${ }^{73}$ Gonzalez R, De-La-Rosa-Ramirez J, Maldonado-Hernandez A et al. Should patients undergoing a bronchoscopy be sedated? Acta Anaesthesiol Scand 2003; 47: $411-415$

${ }^{74}$ Maguire GP, Rubinfield AR, Trembath PW et al. Patients prefer sedation for fiberoptic bronchoscopy. Respirology 1998; 3: 81 - 85

75 Putinati S, Ballerin L, Corbetta L et al. Patient satisfaction with conscious sedation for bronchoscopy. Chest 1999; 115: 1437-1440

${ }^{76}$ Matot I, Kramer MR. Sedation in outpatient flexible bronchoscopy. Alfentanil-Propofol versus Meperidine-Midazolam. J Bronchol 1999; 6: $74-77$

77 Crawford M, Pollock J, Anderson K et al. Comparison of midazolam with propofol for sedation in outpatient bronchoscopy. Br J Anaesth 1993; 70: 419-422

${ }^{78}$ Roffe C, Smith MJ, Basran GS. Anticholinergic premedication for fiberoptic bronchoscopy. Monaldi Arch Chest Dis 1994; 49: 101 - 106

79 Baer G, Annila P. Atropine as premedication for anaesthesia and bronchoscopy. Lancet 1995; 345: 1375

${ }^{80}$ Williams T, Brooks T, Ward C. The role of atropine premedication of fiberoptic bronchoscopy using intravenous midazolam sedation. Chest 1998; 113: $1394-1398$

${ }^{81}$ Cowl CT, Prakash UBS, Kruger BR. The role of anticholinergics in bronchoscopy. A randomized clinical trial. Chest 2000; 118: 188-192

82 Graham PR, Schoder A, Warzelhan J et al. Bronchoskopie und Herzrhythmusveränderungen. Ist eine Prämedikation mit Atropin stets erforderlich? Pneumologie 2002; 56: $593-598$

${ }^{83}$ Makker H, Kishen R, O'Driscoll R. Atropine as premedication for bronchoscopy. Lancet 1995; 345: 724-725

${ }^{84}$ Milman N, Faurschou P, Grode G et al. Pulse oximetry during fiberoptic bronchoscopy in local anaesthesia: frequency of hypoxaemia and effect of oxygen supplementation. Respiration 1994; 61: 342 - 347

${ }^{85}$ Shrader D, Lakshminarayan S. The effect of fiberoptic bronchoscopy on cardiac rhythm. Chest 1978; 73: 821-824

${ }^{86}$ Katz A, Michelson E, Stawicki J et al. Cardiac arrhythmias. Frequency during fiberoptic bronchoscopy and correlation with hypoxemia. Arch Intern Med 1981; 141: 603-606

${ }^{87}$ Evans E, Ganeshalingam K, Ebden P. Changes in oxygen saturation and transcutaneous carbon dioxide and oxygen levels in patients undergoing fiberoptic bronchoscopy. Respir Med 1998; 92: 739- 742

${ }^{88}$ Luck J, Messeder O, Rubenstein M et al. Arrhythmias from fiberoptic bronchoscopy. Chest 1978; 74: 139-143
${ }^{89}$ Breuer $\mathrm{H}$, Charchut S, Worth $\mathrm{H}$. Effects of diagnostic procedures during fiberoptic bronchoscopy on heart rate, blood pressure, and blood gases. Klin Wochenschr 1989; 6: 524-529

${ }^{90}$ Matot I, Kramer M, Glantz L et al. Myocardial ischemia in sedated patients undergoing fiberoptic bronchoscopy. Chest 1997; 112: $1454-1458$

${ }^{91}$ Joyner LR, Scheinhorn DJ. Transbronchial forceps lung biopsy through the fiberoptic bronchoscope. Diagnosis of diffuse pulmonary disease. Chest 1975; 67: $532-535$

92 Puar HS, Young RC, Armstrong EM. Bronchial and transbronchial lung biopsy without fluoroscopy in sarcoidosis. Chest 1985; 87: 303-306

${ }^{93}$ De Fenoyl O, Capron F, Lebeau B et al. Transbronchial biopsy without fluoroscopy: a five year experience in outpatients. Thorax 1989; 44: 956-959

${ }^{94}$ Anders CGT, Johnson MJE, Bush MBA et al. Transbronchial biopsy without fluoroscopy. A seven-year perspective. Chest 1998; 94: $557-560$

${ }^{95}$ Milligan SA, Luce JM, Golden J et al. Transbronchial biopsy without fluoroscopy in patients with diffuse roentgenographic infiltrates and the aquired immunodeficiency syndrome. Am Rev Respir Dis 1988; 137: $486-488$

${ }^{96}$ Gasparini S, Ferretti M, Secchi EB et al. Integration of transbronchial and percutaneous approach in diagnosis of peripheral pulmonary nodules or masses. Experience with 1.027 consecutive cases. Chest 1995; 108: $131-137$

${ }^{97}$ Gupta D, Gulati M, Rajwanshi A. Fluoroscopic transbronchial fine needle aspiration for diagnosis of peripheral pulmonary nodules. Indian J Chest Dis Allied Sci 1996; 38: 163 - 167

${ }^{98}$ Ahmad M, Livingston DR, Golish J et al. The safety of outpatient transbronchial biopsy. Chest 1986; 90: 403-405

${ }^{99}$ Lery H, Kallenbach JM, Feldman C et al. Delayed pneumothorax after transbronchial lung biopsy. Thorax 1986; 41: 647-648

${ }^{100}$ Hernandez BL, Sanchez HiM, Villena GV et al. Safety of the transbronchial biopsy in outpatients. Chest 1991; 99: $562-565$

101 Becker HB, Herth F. Endobronchial ultrasound of the airways and the mediastinum. In: Bolliger CT, Mathur PN (eds): Interventional Bronchoscopy. Basel: Karger Publisher, 2000

102 Falcone F, Fois F, Grosso D. Endobronchial ultrasound. Respiration 2003; 70: 179-194

103 Colt HG. Functional evaluation before and after interventional bronchoscopy. In: Bolliger CT, Mathur PN (eds): Interventional Bronchoscopy. Basel: Karger Publisher, 2000

${ }^{104}$ Kristensen M, Milman N, Jarnoig J. Pulse oximetry at fiberoptic bronchoscopy in local anaesthesia: indication for postbronchoscopy oxygen supplementation? Respir Med 1998; 92: 432-437

105 Kuhn K, Swobodnik W, Johannes RS et al. The quality of gastroenterological reports based on free text dictation: an evaluation in endoscopy and ultrasonography. Endoscopy 1991; 23: 262-264

106 Gouveia-Oliveira A, Raposo VD, Salgado NC et al. Longitudinal compartive study on the influence of computers on reporting of clinical data. Endoscopy 1991; 23: 334-337

107 Mai HD, Sanowski RA, Waring JP. Improved patient care using the ASGE-guidelines on quality assurance: a prospective comparative study. Gastrointest Endoscopy 1991; 37: 597-599

${ }^{108}$ Haponik EF, Russell GB, Beamis JF et al. Bronchoscopy training. Current fellows' experiences and some concerns for the future. Chest 2000; 118: 625-630

${ }^{109}$ Kvale PA, Prakasch UBS. Teaching bronchoscopy. In: Prakash UBS (ed): bronchoscopy. New York: Raven Press, 1994

${ }^{110}$ Neumann M, Hochberger J, Felzmann T et al. Erlanger Endo-Trainer. Endoscopy 2001; 33: 887-890 